\title{
Homogeneous black strings in Einstein-Gauss-Bonnet with Horndeski hair and beyond
}

\author{
Adolfo Cisterna $^{1, \mathrm{a}}$, Sebastián Fuenzalida ${ }^{2, \mathrm{~b}}$, Marcela Lagos ${ }^{2, \mathrm{c}}$, Julio Oliva ${ }^{2, \mathrm{~d}}$ \\ ${ }^{1}$ Centro de Ingeniería y Desarrollo Sustentable, Facultad de Ingeniería, Universidad Central de Chile, Santa Isabel 1186, 8330601 Santiago, Chile \\ ${ }^{2}$ Departamento de Física, Universidad de Concepción, Casilla 160-C Concepción, Chile
}

Received: 5 October 2018 / Accepted: 8 November 2018 / Published online: 28 November 2018

(C) The Author(s) 2018

\begin{abstract}
In this paper we construct new exact solutions in Einstein-Gauss-Bonnet and Lovelock gravity, describing asymptotically flat black strings. The solutions exist also under the inclusion of a cosmological term in the action, and are supported by scalar fields with finite energy density, which are linear along the extended direction and have kinetic terms constructed out from Lovelock tensors. The divergenceless nature of the Lovelock tensors in the kinetic terms ensures that the whole theory is second order. For spherically, hyperbolic and planar symmetric spacetimes on the string, we obtain an effective Wheeler's polynomial which determines the lapse function up to an algebraic equation. For the sake of concreteness, we explicitly show the existence of a family of asymptotically flat black strings in six dimensions, as well as asymptotically $\mathrm{AdS}_{5} \times \mathbb{R}$ black string solutions and compute the temperature, mass density and entropy density. We compute the latter by Wald's formula and show that it receives a contribution from the non-minimal kinetic coupling of the matter part, shifting the one-quarter factor coming from the Einstein term, on top of the usual non areal contribution arising from the quadratic Gauss-Bonnet term. Finally, for a special value of the couplings of the theory in six dimensions, we construct strings that contain asymptotically AdS wormholes as well as rotating solutions on the transverse section. By including more scalars the strings can be extended to $p$-branes, in arbitrary dimensions.
\end{abstract}

\section{Introduction}

Higher dimensional general relativity (GR) possesses a broader spectrum of black hole solutions as compared with

\footnotetext{
a e-mail: adolfo.cisterna@ucentral.cl

be-mail: sfuenzalida@udec.cl

c e-mail: marcelagos@udec.cl

de-mail: julioolivazapata@gmail.cl
}

its four dimensional formulation $[1,2]$. Indeed, four dimensional GR is constrained by uniqueness theorems [3-5] that ensure that any black hole solution of the theory is contained in the Kerr family [6,7]. Moreover, topological restrictions allow only for horizons with spherical topology [8]. The spectrum of solutions is limited not only quantitatively but also qualitatively, setting the final state of black hole collapse to be described only by a small set of parameters [9]. On the other hand it is well-known that gravity in higher dimensions admits spacetimes with horizons that can have more general topologies than that of the $(d-2)$-sphere [1,2], being the existence of black strings, a black hole solution with horizon structure $S^{(d-3)} \times R$, the simplest counterexample [3-5] as it coexists with the Schwarzschild-Tangherlini black hole [10]. Black strings also paved the road for the construction of more sophisticated asymptotically flat solutions with nonspherical topology such as black rings [11] and diverse black object solutions [2], demonstrating how topological restrictions [8] are weakened in higher dimensions. It was shown that black strings are affected by Gregory-Laflamme (GL) instability [12,13], a long-wavelength perturbative instability triggered by a mode that travels along the extended direction of the horizon and, moreover in dimension five the numerical simulations indicate that the instability ends in the formation of naked singularities $[14,15]$, representing an explicit failure of the cosmic censorship in higher dimensions [16]. ${ }^{1}$

Black strings in GR in vacuum are easy to construct. In fact they are obtained by a cylindrical oxidation of the Schwarzschild black hole in $d$ dimensions by the inclusion of $p$ extra flat coordinates. This can be realized by seeing that the equations of motion along the extended directions are compatible with the field equations on the $p$-brane, due to the fact that the involved curvature quantities vanish on these coordinates.

\footnotetext{
1 This feature is also present in the black ring [17] and the Myers-Perry solution in six dimensions [18].
} 
Notwithstanding it is not hard to find simple scenarios in which the construction of analytic black strings fails. Indeed, the mere inclusion of a cosmological constant spoils the existence of cylindrically extended black strings, since the compatibility of the equations of motion on the flat coordinates with the trace of the field equations along the brane forces $\Lambda$ to vanish. This implies that there is no simple oxidation of the Schwarzschild (A)dS black hole. ${ }^{2}$

In Ref. [23] two of the authors have shown that GR with a negative cosmological constant does admit exact, homogeneous black string solutions if each extended flat coordinate is dressed with a massless, minimally coupled scalar field that depends exclusively on that coordinate. ${ }^{3}$ To see this explicitly let us take the following black string ansatz in which a $d$-dimensional black hole is oxidated by including $p$ flat directions

$d s^{2}=-F(r) d t^{2}+\frac{d r^{2}}{F(r)}+r^{2} d \Sigma_{d-2, K}^{2}+\delta_{i j} d x^{i} d x^{j}$,

where $i, j=1,2, \ldots, p$ and $d \Sigma_{d-2, K}^{2}$ stands for a $(d-2)$ dimensional Euclidean manifold of constant curvature $\gamma=$ $0, \pm 1$. By virtue of the ansatz ( 1 ), the massless Klein-Gordon equations deliver scalar fields that depend linearly on the extended flat directions

$\psi_{(j)}\left(x^{j}\right)=\lambda_{(j)} x^{j}, \quad$ no sum over $j$.

the $\lambda_{(j)}$ being integration constants. These scalars cure the incompatibility between the equations of motion on the brane and the extended directions leading to the metric

$F(r)=K-\frac{2 \mu}{r^{d-3}}-\frac{2 \Lambda r^{2}}{(d-1)(d+p-2)}$,

where compatibility is ensured provided

$\lambda_{(j)}^{2}=\lambda^{2}=-\frac{4 \Lambda}{(d+p-2)}$.

This solution represents the black string version of the Schwarzschild-AdS black hole and shows that the threedimensional BTZ black hole can be also uplifted to a higher dimensional black string. ${ }^{4}$

A natural question arises: as higher dimensional objects, do homogeneous and cylindrically extended black strings

\footnotetext{
${ }^{2}$ Numerical as well as perturbative solutions have been constructed in [19-22] in GR as well as in gauged supergravity in five dimensions.

${ }^{3}$ Previously, AdS black strings in GR were constructed considering warped spacetimes [24] providing non-homogenous configurations.

${ }^{4}$ This can also be achieved in the Einstein-Skyrme system [25] and even more by using this approach, diverse BTZ black strings have been recently constructed in theories with non-trivial torsion [26].
}

and black $p$-branes exist in higher curvature extensions of GR?

In this paper we answer this question affirmatively for the Einstein-Gauss-Bonnet as well as for general Lovelock theories with arbitrary values of the coupling constants. To successfully apply the procedure before described, we shall observe that the extended directions must be dressed with scalar fields of the type (2) that have non-minimal kinetic couplings, such that the extra higher curvature terms involved in Lovelock theories do not break the compatibility of the field equations. Such couplings must fulfil the following requirements: They must enjoy shift symmetry, which allows the inclusion of non-minimal kinetic couplings. Secondly, their contribution to the equations of motion must be of the same type as that of the Lovelock terms under consideration. These types of non-minimal kinetic couplings arise naturally in Galileon/Horndeski theory namely, the most general scalartensor theory with second order field equations for both, the metric and the scalar field. Originally constructed in the early seventies, Horndeski theory [27] has re-emerged after the appearances of Galileon theories [28-30] mostly motivated by their several applications in cosmology [31-33]. The Horndeski Lagrangian, which has been constructed in four dimensions, has a non-minimal kinetic sector given by

$\mathcal{L} \sim G_{A B} \partial^{A} \chi \partial^{B} \chi$

where $G_{A B}$ is the Einstein tensor. This term was extensively considered not only in cosmology [34-38] but also in the study of compact objects such as black holes and neutron stars [39-47]. We will see that the higher dimensional generalizations of this term are precisely the kind of couplings we need to consider in order to construct homogeneous black strings in Lovelock gravity. The shift invariant scalars will provide the dress we need in order to ensure compatibility of the equations of motion.

This paper is organized as follows: To fix ideas, Sect. 2 is destined to construct homogeneous black strings in EinsteinGauss-Bonnet theory dressed by two scalar fields, a minimally coupled one accounting for the inclusion of the cosmological constant and a non-minimally coupled one accounting for the new $R^{2}$ curvature terms we are including. We present the equations in arbitrary dimension $D=d+1$, nevertheless for the sake of concreteness we focus on the minimal dimension allowing for new black strings which in this case is $D=(1+4)+1$ and analyze the causal structures as well as the thermal properties of the black strings. Section 3 is devoted to extend the previous results to theories containing arbitrarily higher curvature terms in the Lovelock family. Remarkably, we show that there is a pattern that allows to construct homogeneous AdS black strings in Lovelock theories supported by a family of scalars with generalized non-minimal kinetic terms, leading to a Wheeler's like poly- 
nomial with effective couplings. Assuming the existence of an event horizon, we compute the temperature, as well as the entropy of the black hole. In Sect. 4 we revisit the EinsteinGauss-Bonnet theory and show that for special values of the matter couplings, if the transverse section of the black strings and $p$-branes is five dimensional, the theory allows for wormholes as well as rotating solutions. The latter leads to the first example of a rotating solution in Einstein-GaussBonnet theory in arbitrary dimensions. Finally in Sect. 5 we outline our conclusions and further developments that can follow this work.

\section{Homogeneous black strings in Einstein-Gauss-Bonnet theory}

Let's consider the Einstein-Gauss-Bonnet theory in arbitrary dimension coupled to two scalar fields as follows:

$$
\begin{aligned}
I= & \int d^{D} x \sqrt{-g}\left(R-2 \Lambda_{0}+\alpha \mathcal{L}_{G B}\right. \\
& \left.-\frac{1}{2} g_{A B} \partial^{A} \psi \partial^{B} \psi+\frac{\gamma}{2} G_{A B} \partial^{A} \chi \partial^{B} \chi\right),
\end{aligned}
$$

where $\mathcal{L}_{G B}:=R^{2}-4 R_{A B} R^{A B}+R_{A B C D} R^{A B C D}$ defines the Gauss-Bonnet term. Performing the variations respect to the metric and the scalar fields we obtain the following set of field equations

$$
\begin{aligned}
& G_{A B}+\Lambda_{0} g_{A B}+\alpha H_{A B}=T_{A B}^{(1)}+T_{A B}^{(2)}, \\
& g^{A B} \nabla_{A} \nabla_{B} \psi=0, \\
& \gamma G^{A B} \nabla_{A} \nabla_{B} \chi=0,
\end{aligned}
$$

where the Gauss-Bonnet tensor is given by

$$
\begin{aligned}
H_{A B}= & 2 R R_{A B}-4 R_{A C B D} R^{C D}+2 R_{A C D E} R_{B}^{C D E} \\
& -4 R_{A C} R_{B}{ }^{C}-\frac{1}{2} g_{A B} \mathcal{L}_{G B},
\end{aligned}
$$

and the energy-momentum tensors for the scalars read

$$
\begin{aligned}
T_{A B}^{(1)}= & \frac{1}{2}\left(\partial_{A} \psi \partial_{B} \psi-\frac{1}{2} g_{A B}(\partial \psi)^{2}\right) \\
T_{A B}^{(2)}= & \frac{\gamma}{2}\left(\frac{1}{2} \partial_{A} \chi \partial_{B} \chi R-2 \partial_{C} \chi \partial_{(A} \chi R_{B)}^{C}\right. \\
& -\partial_{C} \chi \partial_{D} \chi R_{A}^{C}{ }_{B}^{D}-\nabla_{A} \nabla^{C} \chi \nabla_{B} \nabla_{C} \chi+\nabla_{A} \nabla_{B} \chi \square \chi \\
& +\frac{1}{2} G_{A B}(\partial \chi)^{2}-g_{A B}\left[-\frac{1}{2} \nabla^{C} \nabla^{D} \chi \nabla_{C} \nabla_{D} \chi\right. \\
& \left.\left.+\frac{1}{2}(\square \chi)^{2}-\partial_{C} \chi \partial_{D} \chi R^{C D}\right]\right) .
\end{aligned}
$$

Note that the Eq. (9) are linear on the scalars.

On a metric of the form

$$
d s^{2}=d \tilde{s}_{d}^{2}+d z^{2}
$$

and scalars that depend only on the extended direction $z$, the equations for the scalars imply a linear dependence on $z$. The shift symmetry of the scalar field theories can be used to set to zero the additive integration constants that appears in both scalars. So we have

$\psi(z)=c_{0} z$ and $\quad \chi(z)=c_{1} z$

with $c_{0}$ and $c_{1}$, integration constants. Then, the trace of the field equations on the $d$-dimensional manifold and the equation along the $z$ direction respectively read

$$
\begin{aligned}
\mathcal{E}_{1}:= & \left(\Lambda_{0}+\frac{c_{0}^{2}}{4}\right) d+\left(1-\frac{\gamma}{4} c_{1}^{2}\right)\left(1-\frac{d}{2}\right) \tilde{R} \\
& +\alpha\left(2-\frac{d}{2}\right) \tilde{\mathcal{L}}_{G B}=0, \\
\mathcal{E}_{2}:= & \left(\Lambda_{0}-\frac{c_{0}^{2}}{4}\right)-\frac{1}{2}\left(1+\frac{\gamma}{4} c_{1}^{2}\right) \tilde{R}-\frac{\alpha}{2} \tilde{\mathcal{L}}_{G B}=0,
\end{aligned}
$$

where $\tilde{R}$ and $\tilde{\mathcal{L}}_{G B}$ are the intrinsic Ricci scalar and GaussBonnet combination of the $d$-dimensional manifold with line element $d \tilde{s}_{d}$. To avoid incompatibilities these two equations must be proportional term by term, i.e. $\mathcal{E}_{1}=\xi \mathcal{E}_{2}$ and therefore, when the higher curvature Gauss-Bonnet term is present, one obtains that the proportionality constant must be fixed as well as the integration constants of the scalars, leading to ${ }^{5}$

$\xi=\frac{1}{d-4}, c_{0}^{2}=-\frac{8 \Lambda_{0}}{d-2}$ and $\quad c_{1}^{2}=\frac{4}{(d-3) \gamma}$.

Under these conditions, we will have a solution of the theory (6) in arbitrary dimension $D=d+1$ for the scalars

$\psi(z)=\sqrt{-\frac{8 \Lambda_{0}}{d-2}} z$ and $\chi(z)=\sqrt{\frac{4}{(d-3) \gamma}} z$

provided the $d$-dimensional metric $d \tilde{s}_{d}^{2}$ fulfils the EinsteinGauss-Bonnet field equations with the following rescaled couplings

$\frac{(d-4)(d-3) \Lambda_{0}}{(d-2)} \tilde{g}_{\mu \nu}+(d-4) \tilde{G}_{\mu \nu}+(d-3) \alpha \tilde{H}_{\mu \nu}=0$

\footnotetext{
5 Note that when $\alpha=0$, one obtains only two equations for the three constant $\xi, c_{0}$ and $c_{1}$, leading to $c_{0}^{2}=-\frac{4 \Lambda_{0}(\xi d-1)}{\xi d+1}$ and $c_{1}^{2}=$ $\frac{4}{\gamma} \frac{((d-2) \xi-1)}{((d-2) \xi+1)}$ for an arbitrary $\xi$. Since we are interested in the inclusion of higher derivative terms, we do not elaborated further in the case $\alpha=0$.
} 
Here $\tilde{g}_{\mu \nu}, \tilde{G}_{\mu \nu}$ and $\tilde{H}_{\mu \nu}$ are, respectively, the intrinsic metric, Einstein tensor and Gauss-Bonnet tensor for the $d$-dimensional metric $d \tilde{s}_{d}^{2}$ on the constant $z$ section of the black string ${ }^{6}$ (10). Since $\tilde{H}_{\mu \nu}$ vanishes identically in dimensions $d \leq 4$, to have a non-vanishing contribution from the Gauss-Bonnet tensor, we need $d \geq 5$ (in $d=4$ the equation (16) is identically fulfilled for any metric and the system is degenerate at such point).

For simplicity, let's focus on the six dimensional case and consider the homogeneous black string metric

$d s_{6}^{2}=-f(r) d t^{2}+\frac{d r^{2}}{f(r)}+r^{2} d \Sigma_{3, K}^{2}+d z^{2}$,

where $d \Sigma_{3, K}$ is the line element of an Euclidean, threedimensional constant curvature manifold of curvature $K$, and assume that the scalars depend only on the $z$ direction.

The explicit $z$-dependence of the scalars is fixed by the scalar equations to be linear, giving rise to

$\psi(z)=\sqrt{-\frac{8 \Lambda_{0}}{3}} z$ and $\chi(z)=\sqrt{\frac{2}{\gamma}} z$

and the metric function has to solve the following quadratic, polynomial equation

$36 \alpha(K-f(r))^{2}+r^{2}\left(9 K-\Lambda_{0} r^{2}-9 f(r)\right)=\frac{6 m}{V_{3}^{(K)}}$

where $m$ is an integration constant and $V_{3}^{(K)}$ stands for the volume of manifold with line element $d \Sigma_{K, 3}$.

For vanishing cosmological term in the action $\left(\Lambda_{0}=0\right)$, the minimally coupled scalar vanishes and as a solution of the polynomial equation one obtain

$f(r)=K+\frac{r^{2}}{8 \alpha}\left(1 \pm \sqrt{1+\frac{32}{3 V_{3}^{(K)}} \frac{m \alpha}{r^{4}}}\right)$.

Consequently, for the spherically symmetric case, the metric describes an asymptotically flat, homogeneous black string, with a regular horizon, in the six-dimensional EinsteinGauss-Bonnet theory supported by a scalar field with an Einstein-kinetic term. These are the first known asymptotically flat, analytic and homogeneous black string solutions in general relativity with a Gauss-Bonnet term.

\footnotetext{
${ }^{6}$ Note that the equations that determine the geometry (16) do not depend on $\gamma$, since the energy-momentum tensor is proportional to the coupling $\gamma$ and at the same time is quadratic in the field $\chi \sim \gamma^{-1 / 2}$.
}

Considering a non-vanishing bare cosmological term, $\Lambda_{0} \neq 0$, the solutions of the polynomial reads

$f(r)=K+\frac{r^{2}}{8 \alpha}\left(1 \pm \sqrt{1+\frac{16}{9} \alpha \Lambda_{0}+\frac{32}{3 V_{3}^{(K)}} \frac{m \alpha}{r^{4}}}\right)$.

As expected, this has the form of the topological extension of the Boulware-Deser metric with a cosmological constant [48].

From the expression (18) we see that the bare cosmological constant must fulfil $\Lambda_{0} \leq 0$, therefore, for simplicity and without loosing generality, let us set

$\Lambda_{0}=-1$.

For positive values of the Gauss-Bonnet coupling $(\alpha>0)$ and spherically symmetric spacetimes on the brane $(K=1)$ only the branch with the negative sign in (21) might lead to an event horizon. In this case, the asymptotic behavior of the metric is

$$
\begin{aligned}
f(r) & =1+\frac{r^{2}}{8 \alpha}\left(1-\sqrt{1-\frac{16}{9} \alpha}\right)+\mathcal{O}\left(M r^{-2}\right) \\
& =1+\frac{r^{2}}{l^{2}}+\mathcal{O}\left(M r^{-2}\right)
\end{aligned}
$$

which induces an upper bound on the Gauss-Bonnet coupling $0<\alpha<\frac{9}{16}$, where we have defined

$\frac{1}{l^{2}}=\frac{1}{8 \alpha}\left(1-\sqrt{1-\frac{16}{9} \alpha}\right)$.

Then, the asymptotic behavior of the metric on the brane is that of the $A d S_{5}$ spacetime and the subleading term is controlled by the integration constant $m$, leading to a finite mass contribution (mass density if one considers the extended direction). Considering $\alpha$ positive and $l^{2}$ to be the curvature radius of the brane at infinity, leads to the restriction $\frac{9}{2}<$ $l^{2}<9$.

Wald's formula $[49,50]$ can be used to obtain the following entropy per unit length of the string

$s=4 \pi^{3} r_{+}^{3}+96 \pi^{3} r_{+} \alpha$,

where $r_{+}$is the horizon radius. Here we note that the Einstein contribution to the entropy receives a correction from the matter sector. Since in this case the Bekenstein-Hawking entropy should be $\frac{A}{4 G}=8 \pi^{3} r_{+}^{3} L$ (with $16 \pi G=1$ ). As expected on dimensional grounds, the term proportional to the Gauss-Bonnet coupling depends linearly on the horizon radius. As occurs for black holes, the entropy of the planar 
horizons do not receive corrections from the higher curvature terms.

The temperature is fixed in order to obtain a smooth Euclidean continuation on the horizon and is given by

$T=\frac{2 r_{+}^{3}+9 r_{+}}{2 \pi\left(9 r_{+}^{2}-2 l^{4}+18 l^{2}\right)}$.

One can see that, identifying the integration constant $m$ with the mass density, the thermodynamical quantities fulfil the first law

$d m=T d s$.

Since the integration constants of the scalars are fixed in terms of the couplings, it is natural to expect that the first law does not receive contributions from the matter sector. A plot with different profiles for the function $f(r)$ is presented in Fig. 1, for both the asymptotically flat (left panel) and asymptotically AdS solutions (right panel).

We have therefore shown that the inclusion of the nonminimal Einstein-kinetic coupling (5) allows to construct black strings in Einstein-Gauss-Bonnet theory, for arbitrary values of the coupling constants of the theory.

As mentioned above, the obstruction to the existence of cylindrically oxidated solutions comes from the incompatibility between the trace of the field equations on the brane and the equation along the extended direction. The scalar fields $\psi$ and $\chi$ provide a natural manner to circumvent this incompatibility even in the asymptotically flat case. Below, we give a detailed explanation of the mechanism behind the existence of these solutions for general Lovelock theories, and show that the results can be extended beyond EinsteinGauss-Bonnet by the inclusion of scalars with non-minimal kinetic coupling to Lovelock tensors, that naturally extend (5).

\section{Homogeneous black strings in general Lovelock theory}

Let us supplement the Lovelock action by quadratic scalars in the following form

$$
I\left[g_{A B}, \phi_{(k)}\right]=\int d^{D} x\left[\sum_{k=0}^{n+1} \alpha_{k} \mathcal{L}^{(k)}+\sum_{k=0}^{n} \beta_{k} \mathcal{L}_{\text {matter }}^{(k)}\right],
$$

where the Lovelock Lagrangians are given by

$\mathcal{L}^{(k)}=\frac{\sqrt{-g}}{2^{k}} \delta_{B_{1} \cdots B_{2 k}}^{A_{1} \cdots A_{2 k}} R^{B_{1} B_{2}}{ }_{A_{1} A_{2}} \cdots R^{B_{2 k-1} B_{2 k}} A_{2 k-1} A_{2 k}$, and each of the matter fields $\phi_{(k)}=\{\psi, \chi, \ldots\}$ with $k=0,1, \ldots, n$, have different dynamics controlled by the Lagrangian

$$
\begin{aligned}
\mathcal{L}_{\text {matter }}^{(k)}= & \frac{\sqrt{-g}}{2^{k}} E_{C D}^{(k)} \nabla^{C} \phi_{(k)} \nabla^{D} \phi_{(k)} \\
= & -\frac{\sqrt{-g}}{2^{2 k+1}} \nabla_{C} \phi_{(k)} \nabla^{D} \phi_{(k)} \delta_{D B_{1} \cdots B_{2 k}}^{C A_{1} \cdots A_{2 k}} R^{B_{1} B_{2}} A_{1} A_{2} \\
& \cdots R^{B_{2 k-1} B_{2 k}} A_{2 k-1} A_{2 k},
\end{aligned}
$$

which couples the $k$ th scalar directly to the $k$-th order Lovelock tensor $E_{C D}^{(k)}$ (33). The Lovelock tensor has a divergence that vanishes identically since it corresponds to the EulerLagrange derivative with respect to the metric of a diffeomorphism invariant action. The $\alpha_{k}$ are the dimensionful Lovelock couplings and $\beta_{k}$ are the matter couplings.

Varying (29) with respect metric $g^{A B}$ we obtain the field equations

$\mathcal{E}_{A B}:=\sum_{k=0}^{n+1} \alpha_{k} E_{A B}^{(k)}-\sum_{k=0}^{n} \frac{\beta_{k}}{2^{2 k+1}} T_{A B}^{(k)}=0$,

with the Lovelock tensor of order $k$ defined as

$$
\begin{aligned}
E_{A B}^{(k)}:= & -\frac{1}{2^{k+1}} g_{(A \mid C} \delta_{\mid B) B_{1} \cdots B_{2 k}}^{C A_{1} \cdots A_{2 k}} R^{B_{1} B_{2}} A_{1} A_{2} \\
& \cdots R^{B_{2 k-1} B_{2 k}} A_{2 k-1} A_{2 k} .
\end{aligned}
$$

Furthermore, the energy-momentum tensor associated with the $k$ th scalar $\phi_{(k)}$ is given by

$$
\begin{aligned}
& T_{A B}^{(k)}=-\frac{1}{2} g_{A B} \nabla_{C} \phi_{(k)} \nabla^{D} \phi_{(k)} \delta_{D B_{1} \cdots B_{2 k}}^{C A_{1} \cdots A_{2 k}} R^{B_{1} B_{2}} A_{1} A_{2} \\
& \cdots R^{B_{2 k-1} B_{2 k}} A_{2 k-1} A_{2 k} \\
& +\nabla_{C} \phi_{(k)} \nabla_{(A \mid} \phi_{(k)} \delta_{\mid B) B_{1} \cdots B_{2 k}}^{C A_{1} \cdots A_{2 k}} R^{B_{1} B_{2}} A_{1} A_{2} \\
& \cdots R^{B_{2 k-1} B_{2 k}} A_{2 k-1} A_{2 k} \\
& +k \nabla_{C} \phi_{(k)} \nabla^{D} \phi_{(k)} \delta_{D B_{1} \cdots(A \mid}^{C A_{1} \cdots A_{2 k}} R^{B_{1} B_{2}} A_{1} A_{2} \\
& \left.\cdots R^{B_{2 k-3} B_{2 k-2}} A_{2 k-3} A_{2 k-2} R^{B_{2 k-1}} \mid B\right) A_{2 k-1} A_{2 k} \\
& +2 k \nabla^{B_{2 k-1}} \nabla_{C} \phi_{(k)} \nabla_{A_{2 k-1}} \nabla^{D} \phi_{(k)} \delta_{D B_{1} \cdots(B \mid}^{C A_{1} \cdots A_{2 k}} R^{B_{1} B_{2}} A_{1} A_{2} \\
& \cdots R^{B_{2 k-3} B_{2 k-2}} A_{2 k-3} A_{2 k-2} g_{\mid A) A_{2 k}} \\
& +k \nabla_{C} \phi_{(k)} \nabla^{E} \phi_{(k)} \delta_{D B_{1} \cdots(B \mid}^{C A_{1} \cdots A_{2 k}} R^{B_{1} B_{2}} A_{1} A_{2} \\
& \cdots R^{B_{2 k-3} B_{2 k-2}} A_{2 k-3} A_{2 k-2} R_{A_{2 k-1} E^{B_{2 k-1} D_{g}} g_{\mid A) A_{2 k}}} \text {. }
\end{aligned}
$$

On the other hand, by varying the action with respect to field $\phi_{(k)}$, we obtain the field equations

$\beta_{k} E_{A B}^{(k)} \nabla^{A} \nabla^{B} \phi_{(k)}=0$ with $k=0, \ldots, n$.

Below we will show that these equations admit homogeneous black strings by requiring the trace of the equations on the 
Existence of event horizons for different values of $m$

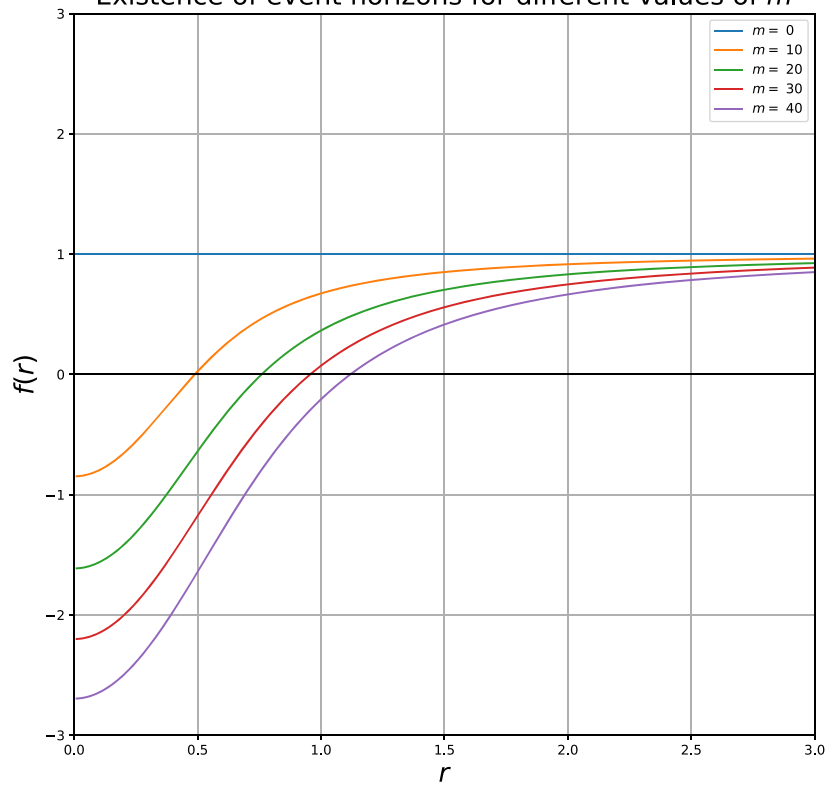

Fig. 1 Here we plot the metric function $f(r)$ coming from the effective Wheeler's polynomial, for different values of the integration constant $m$. The black holes in the left panel lead to asymptotically flat black strings in Einstein-Gauss-Bonnet theory in six dimensions, while the

brane to be compatible with the equation along the extended direction.

We consider the ansatz

$d s^{2}=d \tilde{s}_{d}^{2}+d z^{2}$

where

$d \tilde{s}_{d}^{2}=-f(r) d t^{2}+\frac{d r^{2}}{f(r)}+r^{2} d \Sigma_{K, d-2}^{2}$,

and the scalar fields depending only on the extended coordinate. The equations for the fields (35) imply that the scalars are linear in the extended direction, and the invariance under constant shifts of the matter actions can be used to write

$\phi_{(k)}=c_{(k)} z$.

Here $d \Sigma_{K, d-2}$ denotes the line element of an Euclidean $(d-2)$-dimensional manifold with constant curvature $K= \pm 1,0$.

We will split the $D$-dimensional indices $\{A, B, C, \ldots\}$ in $\{\mu, v, \ldots\}$ that run on the brane with line element $d \tilde{s}_{d}$ and $z$ along the extended direction. Quantities intrinsically defined on the $d$-dimensional manifold will have tildes on top.

To explain the previously mentioned compatibility, we will be as explicit as possible in what follows. The field Eq. (32) read
Existence of event horizons for different values of $m$

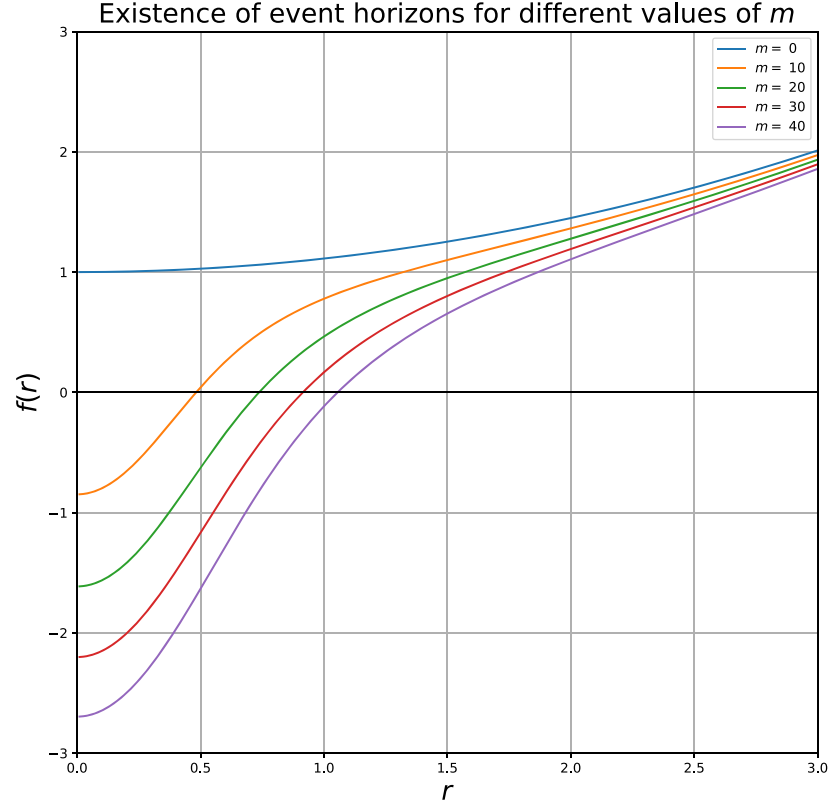

right panel lead to asymptotically $A d S_{5} \times R$ black strings. Note that the metric function $f(r)$ is regular at the origin, as it occurs for EGB in five dimensions in vacuum

$$
\begin{aligned}
\alpha_{0} & E^{(0) A}{ }_{B}+\alpha_{1} E^{(1) A}{ }_{B}+\alpha_{2} E_{B}^{(2) A}+\alpha_{3} E_{B}^{(3) A}{ }_{B} \\
& +\cdots+\alpha_{n+1} E^{(n+1) A}{ }_{B} \\
= & \frac{\beta_{0}}{2} T^{(0) A}{ }_{B}+\frac{\beta_{1}}{8} T^{(1) A}{ }_{B}+\frac{\beta_{2}}{32} T^{(2) A}{ }_{B} \\
& +\cdots+\frac{\beta_{n}}{2^{2 n+1}} T^{(n) A}{ }_{B} .
\end{aligned}
$$

Then, introducing (36) and (38) into equation (39), we obtain the equations with free indices on the string $\mathcal{E}_{\mu \nu}=0$ which imply

$$
\begin{aligned}
& -\frac{\tilde{\alpha}_{0}}{2} \delta_{v}^{\mu}-\frac{\tilde{\alpha}_{1}}{4} \delta_{v \beta_{1} \beta_{2}}^{\mu \alpha_{1} \alpha_{2}} \tilde{R}^{\beta_{1} \beta_{2}} \alpha_{1} \alpha_{2} \\
& -\frac{\tilde{\alpha}_{2}}{8} \delta_{v \beta_{1} \cdots \beta_{4}}^{\mu \alpha_{1} \cdots \alpha_{4}} \tilde{R}_{\alpha_{1} \alpha_{2}}^{\beta_{1} \beta_{2}} \tilde{R}_{\beta_{3} \beta_{4} \alpha_{4}}^{\beta_{2}-\cdots} \\
& -\frac{\tilde{\alpha}_{n}}{2^{n+1}} \delta_{\nu \beta_{1} \cdots \beta_{2 n}}^{\mu \alpha_{1} \cdots \alpha_{2 n}} \tilde{R}_{\alpha_{1} \alpha_{2}}^{\beta_{1} \beta_{2}} \cdots \tilde{R}_{\alpha_{2 n-1} \alpha_{2 n}}^{\beta_{2 n-1} \beta_{2 n}} \\
& -\frac{\tilde{\alpha}_{n+1}}{2^{n+2}} \delta_{\nu \beta_{1} \cdots \beta_{2 n+2}}^{\mu \alpha_{1} \cdots \alpha_{2 n+2}} \tilde{R}_{\alpha_{1} \alpha_{2}}^{\beta_{1} \beta_{2}} \tilde{R}_{\alpha_{2 n+1} \alpha_{2 n+2}}^{\beta_{2 n+1} \beta_{2 n+2}}=0 \text {, }
\end{aligned}
$$

and the equation along the $z$ direction $\mathcal{E}_{z z}$ reads

$$
\begin{aligned}
& -\frac{\hat{\alpha}_{0}}{2}-\frac{\hat{\alpha}_{1}}{4} \delta_{\beta_{1} \beta_{2}}^{\alpha_{1} \alpha_{2}} \tilde{R}^{\beta_{1} \beta_{2}}{ }_{\alpha_{1} \alpha_{2}} \\
& -\frac{\hat{\alpha}_{2}}{8} \delta_{\beta_{1} \cdots \beta_{4}}^{\alpha_{1} \cdots \alpha_{4}} \tilde{R}^{\beta_{1} \beta_{2}}{ }_{\alpha_{1} \alpha_{2}} \tilde{R}^{\beta_{3} \beta_{4}}{ }_{\alpha_{3} \alpha_{4}}-\cdots \\
& -\frac{\hat{\alpha}_{l}}{2^{l+1}} \delta_{\beta_{1} \cdots \beta_{2 n}}^{\alpha_{1} \cdots \alpha_{2 n}} \tilde{R}^{\beta_{1} \beta_{2}}{ }_{\alpha_{1} \alpha_{2}} \cdots \tilde{R}^{\beta_{2 n-1} \beta_{2 n}}{ }_{\alpha_{2 n-1} \alpha_{2 n}}
\end{aligned}
$$




$$
-\frac{\hat{\alpha}_{n+1}}{2^{n+2}} \delta_{\beta_{1} \cdots \beta_{2 n+2}}^{\alpha_{1} \cdots \alpha_{2 n+2}} \tilde{R}_{\alpha_{1} \alpha_{2}}^{\beta_{1} \beta_{2}} \cdots \tilde{R}_{\alpha_{2 n+1} \alpha_{2 n+2}}^{\beta_{2 n+1} \beta_{2 n+2}}=0 .
$$

Here we have introduced for simplicity both sets of shifted gravitational couplings $\tilde{\alpha}_{k}$ and $\hat{\alpha}_{k}$, given respectively by

$\tilde{\alpha}_{k}=\left\{\begin{array}{cc}\alpha_{k}-\frac{\beta_{k}}{2^{k+1}} c_{k}^{2} & \text { for } 0 \leq k \leq n \\ \alpha_{k} & \text { for } k=n+1\end{array}\right.$,

and

$\hat{\alpha}_{k}=\left\{\begin{array}{cc}\alpha_{k}+\frac{\beta_{k}}{2^{k+1}} c_{k}^{2} & \text { for } 0 \leq k \leq n \\ \alpha_{k} & \text { for } k=n+1\end{array}\right.$.

In general, the compatibility of the equations $\tilde{g}^{\mu \nu} \mathcal{E}_{\mu \nu}=0$ and $\mathcal{E}_{z z}=0$ will induce further restrictions on the metric function $f(r)$ than those that come only from the equations on the brane $\mathcal{E}_{\mu \nu}=0$. We can circumvent this clash by imposing the off-shell requirement $\tilde{g}^{\mu \nu} \mathcal{E}_{\mu \nu} \sim \mathcal{E}_{z z}$ and introducing a proportionality constant $\xi$ one obtains

$\hat{\alpha}_{k}=\xi(d-2 k) \tilde{\alpha}_{k}$ for $0 \leq k \leq n$,

and

$\xi(d-2(n+1))=1$.

These equations allow to fix the proportionality constant $\xi$ as well as the $n$ integration constants $c_{k}$ for all the scalars in the following manner

$c_{k}^{2}=\frac{2^{k+1}(n+1-k)}{(d-k-n-1)} \frac{\alpha_{k}}{\beta_{k}} \quad$ with $\quad 0 \leq k \leq n$.

Then, the equations on the brane, reduce to an effective Wheeler-like polynomial that reads

$$
\begin{aligned}
m= & (d-2) V_{d-2}^{(K)} \sum_{k=0}^{n+1} \frac{(d-2 n-2)}{(d-k-n-1)} \frac{(d-3) !}{(d-2 k-1) !} \\
& \times \alpha_{k} r^{d-2 k-1}(K-f(r))^{k} .
\end{aligned}
$$

where $m$ is an integration constant and $V_{d-2}^{(K)}$ stands for the volume of manifold with line element $d \Sigma_{K, d-2}$. This leads to a black string in a general Lovelock theory of gravity that can be asymptotically flat or asymptotically AdS depending of whether we include a cosmological term in the action. The temperature and the entropy of the corresponding black hole are respectively given by
$T=\frac{1}{4 \pi} \frac{\sum_{k=0}^{n+1} \frac{(d-2 k-1)(d-2 n-2)}{(d-k-n-1)} \frac{(d-3) !}{(d-2 k-1) !} \alpha_{k} r_{+}^{d-2 k-2} K^{k}}{\sum_{p=0}^{n+1} \frac{p(d-2 n-2)}{(d-p-n-1)} \frac{(d-3) !}{(d-2 p-1) !} \alpha_{p} r_{+}^{d-2 p-1} K^{p-1}}$.

and

$$
\begin{aligned}
s= & 4 \pi \sum_{k=0}^{n+1} \frac{k(d-2 n-2)}{(d-k-n-1)} \frac{(d-2)}{(d-2 k)} \frac{(d-3) !}{(d-2 k-1) !} \\
& \times \alpha_{k} r_{+}^{d-2 k} K^{k-1} V_{d-2}^{(K)} .
\end{aligned}
$$

and it can be checked that the first law $d m=T d s$ is fulfilled, which lead to the interpretation of the integration constant $m$ as the mass density.

The compatibility of the equations $\tilde{g}^{\mu \nu} \mathcal{E}_{\mu \nu}=0$ and $\mathcal{E}_{z z}=$ 0 is ensured for a general metric on the transverse section of the string, and the scalars

$\phi_{(k)}(z)=\left(\frac{2^{k+1}(n+1-k)}{(d-k-n-1)} \frac{\alpha_{k}}{\beta_{k}}\right)^{1 / 2} z$,

allow to cylindrically extend any solution of Lovelock theory from dimension $d$ to dimension $d+1$. This will be exploited in the next section.

\section{Extra physically interesting solutions}

As usual in Lovelock theories, from the polynomial equation (47) when the integration constant $m$ vanishes, one will obtain $n$ different solutions for the metric function $f(r)$, leading to $n$ different constant curvature solutions on the transverse section of the string. When the curvature radii of these solutions coincide, it is natural to expect an enlargement of the space of solutions of the theory, as it occurs in vacuum. To see this in a concrete example, let us revisit the Einstein-Gauss-Bonnet theory in six dimensions

$$
\begin{aligned}
I= & \int d^{6} x \sqrt{-g}\left(R-2 \Lambda_{0}+\alpha \mathcal{L}_{G B}\right. \\
& \left.-\frac{1}{2} g_{A B} \partial^{A} \psi \partial^{B} \psi+\frac{\gamma}{2} G_{A B} \partial^{A} \chi \partial^{B} \chi\right) .
\end{aligned}
$$

This theory admits a cylindrically extended wormhole solution with line element given by

$d s^{2}=l^{2}\left[-\cosh ^{2}\left(\rho-\rho_{0}\right) d t^{2}+d \rho^{2}+\cosh ^{2} \rho d \Sigma_{3}^{2}\right]+d z^{2}$,

dressed by the scalars

$\psi(z)=\frac{2 \sqrt{3}}{l} z$ and $\chi(z)=\sqrt{\frac{2}{\gamma}} z$, 
provided

$l^{2}=8 \alpha$,

and

$\Lambda_{0} \alpha=-\frac{9}{16}$

The metric (52) describes the cylindrical extension of a wormhole metric, where $\rho_{0}$ is an arbitrary integration constant and $d \Sigma_{3}$ is the line element of an Euclidean, three dimensional space with Ricci scalar equals to -6 [51]. In the original five-dimensional formulation, the wormhole solution [51] exist for Einstein-Gauss-Bonnet theory in the Chern-Simons case in which the five dimensional theory, when formulated in first order formalism, have an enlargement of the local symmetry group from the usual Lorentz group to an AdS local group, and the vielbein and spin connections belong to an AdS gauge connection [52]. From the point of view of a dimensional reduction, the scalars considered here, allow to start with a generic six-dimensional theory and obtained an effective Chern-Simons theory in dimension five.

It is interesting to notice that even though the latter solution has a simple warped structure in five dimensions, one can also embed in a similar manner, a rotating solution of the effective five dimensional Chern-Simons theory, into dimension six. Explicitly the following metric is a solution of the field equations:

$d s^{2}=d \bar{s}_{L}^{2}+\left(\frac{1}{L^{2}}-\frac{1}{l^{2}}\right) \rho^{2}(r, \mu) k_{\alpha} k_{\beta} d x^{\alpha} d x^{\beta}+d z^{2}$,

where $d \bar{s}_{L}$ is the line element of $\mathrm{AdS}_{5}$ with curvature radius $L$, in double oblate coordinates, i.e.

$$
\begin{aligned}
d \bar{s}_{L}^{2}= & -\left(1+\frac{r^{2}}{L^{2}}\right) \frac{\Delta(\mu)}{\Xi_{a} \Xi_{b}} d t^{2}+\frac{r^{2} \rho^{2} d r^{2}}{\left(1+\frac{r^{2}}{L^{2}}\right)\left(r^{2}+a^{2}\right)\left(r^{2}+b^{2}\right)} \\
& +\frac{\rho^{2} d \mu^{2}}{\Delta(\mu)\left(1-\mu^{2}\right)} \\
& +\frac{\left(r^{2}+a^{2}\right)\left(1-\mu^{2}\right)}{\Xi_{a}} d \phi^{2}+\frac{\left(r^{2}+b^{2}\right) \mu^{2}}{\Xi_{b}} d \psi^{2},
\end{aligned}
$$

and $k=k_{\alpha} d x^{\alpha}$ reads

$$
\begin{aligned}
k= & \frac{\Delta(\mu)}{\Xi_{a} \Xi_{b}} d t+\frac{r^{2} \rho^{2} d r}{\left(1+\frac{r^{2}}{L^{2}}\right)\left(r^{2}+a^{2}\right)\left(r^{2}+b^{2}\right)} \\
& +\frac{a\left(1-\mu^{2}\right)}{\Xi_{a}} d \phi+\frac{b \mu^{2}}{\Xi_{b}} d \psi,
\end{aligned}
$$

where $\Xi_{a}=1-\frac{a^{2}}{L^{2}}, \quad \Xi_{b}=1-\frac{b^{2}}{L^{2}}, \Delta(\mu)=\Xi_{a} \mu^{2}+$ $\Xi_{b}\left(1-\mu^{2}\right)$, and $\rho^{2}(r, \mu)=r^{2}+a^{2} \mu^{2}+b^{2}\left(1-\mu^{2}\right)$. The scalar fields are given by (53) and the relations (54) and (55), hold. Note that $l$ is a constant defined by the theory, while $L$ is an integration constant as well as $a$ and $b$. The latter are the socalled oblateness parameters. Since the vector field $k$ defines a null and geodesic congruence of the background metric $d \bar{s}_{L}$, the metric (56) is a cylindrical oxidation of a Kerr-Schild metric in $\mathrm{AdS}_{5}$. The five dimensional metric in the section of the string defines the first known exact, analytic, rotating solution of Einstein-Gauss-Bonnet in five dimensions and it was originally found in [53]. It was also latter proven to be of the non-circular type in [54]. ${ }^{7}$

\section{Final remarks}

Until now in higher curvature gravity, exact and homogeneous black string solutions have been constructed only for special values of the coupling constants $[56,57]$ also including $p$-form fields [58], and the general problem of the construction of Lovelock branes was studied in [59], and for arbitrary values of the couplings only numerical or perturbative solutions were available (see e.g. [60,61]). In this paper we have constructed new, exact, homogeneous black strings in arbitrary Lovelock theories. The solutions are supported by scalar fields with non-minimal kinetic couplings constructed with Lovelock tensors, ensuring that the field equations are of second order. These scalars have been previously considered as part of the higher dimensional extension of Horndeski theories in $[62,63]$. Here, the scalars being dependent only on the coordinate along the extended direction, turn out to be linear, and the proportionality constant gets fixed by the requiring the compatibility of the whole system. The transverse section of the string can be asymptotically flat or $A d S$. For concreteness we computed the thermodynamic quantities and showed that the entropy of the black string receives a contribution from the matter part. This is interesting because the pattern of transitions between black string and black hole can change due to the presence of the new Horndeski fields. The gravitational stability of the black string and $p$-branes in the presence of a single quadratic or cubic Lovelock term has been studied in [64-66], while the effect of quartic corrections coming from $\mathrm{M}$-theory have been explored in [67]. It is interesting to mention that the Large D approach [68] allows to keep all the terms in the Einstein-Gauss-Bonnet Lagrangian [69].

For simplicity we focus on the case with a single extended direction, but this construction also works with $p$-branes. For example, in the Einstein-Gauss-Bonnet theory, with a cos-

\footnotetext{
${ }^{7}$ For a recent extension of the rotating metric in Chern-Simons theories in odd dimensions, within the Lovelock family in vacuum, see [55].
} 
mological constant, one would have to consider $p$ minimally coupled scalars mimicking such of reference [23], as well as $p$ scalars with Einstein-kinetic couplings which would also turn out to be linear and depending on a single extended direction. From this, the extension to arbitrary Lovelock theories, with flat $p$-branes is clear.

Interpreting our solutions as compactifications with nontrivial scalar fluxes along the extended direction, one obtains an effective Lovelock theory induced on the brane. We exploited this idea to construct cylindrically extended solution with wormholes on the transverse section, which are asymptotically $\mathrm{AdS}_{5} \times R$, in both directions. Within the same realm we also constructed a cylindrical oxidation of the rotating spacetime of Einstein-Gauss-Bonnet gravity, constructed from a Kerr-Schild ansatz in [53]. The wormhole solution can of course be extended to $\operatorname{AdS}_{2 n-1} \times R$ provided one considers Lovelock theory with all the possible terms in dimension $2 n$. In such case, the wormhole on the string will be the one reported in $[51,70]$.

Acknowledgements The authors would like to acknowledge E. Babichev, C. Charmousis, N. Grandi and J. Rocha for valuable comments and remarks. J.O. is partially supported by FONDECYT Grant 1181047. A. C. is supported by Fondo Nacional de Desarrollo Científico y Tecnológico Grant no. 11170274 and Proyecto Interno Ucen $I+$ D-2016, CIP2016.

Open Access This article is distributed under the terms of the Creative Commons Attribution 4.0 International License (http://creativecomm ons.org/licenses/by/4.0/), which permits unrestricted use, distribution, and reproduction in any medium, provided you give appropriate credit to the original author(s) and the source, provide a link to the Creative Commons license, and indicate if changes were made.

Funded by SCOAP ${ }^{3}$.

\section{References}

1. N.A. Obers, Lect. Notes Phys. 769, 211 (2009). arXiv:0802.0519 [hep-th]

2. G.T. Horowitz (ed.), Black holes in higher dimensions (Cambridge University Press, Cambridge, 2012)

3. B. Carter, Phys. Rev. Lett. 26, 331 (1971). https://doi.org/10.1103/ PhysRevLett.26.331

4. W. Israel, Commun. Math. Phys. 8, 245 (1968). https://doi.org/10. 1007/BF01645859

5. R.M. Wald, Phys. Rev. Lett. 26, 1653 (1971). https://doi.org/10. 1103/PhysRevLett.26.1653

6. R.P. Kerr, Phys. Rev. Lett. 11, 237 (1963)

7. G.C. Debney, R.P. Kerr, A. Schild, J. Math. Phys. 10, 1842 (1969)

8. J. L. Friedman, K. Schleich and D. M. Witt, Phys. Rev. Lett. 71, 1486 (1993) Erratum: [Phys. Rev. Lett. 75, 1872 (1995)]. https://doi.org/10.1103/PhysRevLett.75.1872, https://doi.org/10. 1103/PhysRevLett.71.1486. arXiv:gr-qc/9305017

9. R. Ruffini, J.A. Wheeler, Phys. Today 24(1), 30 (1971)

10. F.R. Tangherlini, Nuovo Cimento 27, 636 (1963). https://doi.org/ 10.1007/BF02784569

11. R. Emparan, H.S. Reall, Phys. Rev. Lett 88, 101101 (2002). https:// doi.org/10.1103/PhysRevLett.88.101101. arXiv:hep-th/0110260
12. R. Gregory, R. Laflamme, Phys. Rev. Lett 70, 2837 (1993). https:// doi.org/10.1103/PhysRevLett.70.2837. arXiv:hep-th/9301052

13. R. Gregory, R. Laflamme, Nucl. Phys. B 428, 399 (1994). https:// doi.org/10.1016/0550-3213(94)90206-2. arXiv:hep-th/9404071

14. M.W. Choptuik, L. Lehner, I. Olabarrieta, R. Petryk, F. Pretorius, H. Villegas, Phys. Rev. D 68, 044001 (2003). https://doi.org/10. 1103/PhysRevD.68.044001. arXiv:gr-qc/0304085

15. L. Lehner, F. Pretorius, Phys. Rev. Lett. 105, 101102 (2010). https:// doi.org/10.1103/PhysRevLett.105.101102. arXiv:1006.5960 [hepth]

16. R. M. Wald, ed., Black holes and relativistic stars (1998)

17. O.J.C. Dias, P. Figueras, R. Monteiro, J.E. Santos, R. Emparan, Phys. Rev. D 80, 111701 (2009). arXiv:0907.2248 [hep-th]

18. O.J.C. Dias, P. Figueras, R. Monteiro, H.S. Reall, J.E. Santos, JHEP 1005, 076 (2010). arXiv:1001.4527 [hep-th]

19. K. Copsey, G.T. Horowitz, JHEP 0606, 021 (2006). https://doi.org/ 10.1088/1126-6708/2006/06/021. arXiv:hep-th/0602003

20. R.B. Mann, E. Radu, C. Stelea, JHEP 0609, 073 (2006). https:// doi.org/10.1088/1126-6708/2006/09/073. arXiv:hep-th/0604205

21. A. Bernamonti, M.M. Caldarelli, D. Klemm, R. Olea, C. Sieg, E. Zorzan, JHEP 0801, 061 (2008). https://doi.org/10.1088/ 1126-6708/2008/01/061. arXiv:0708.2402 [hep-th]

22. P. Kanti, T. Nakas, N. Pappas, Phys. Rev. D 98(6), 064025 (2018). https://doi.org/10.1103/PhysRevD.98.064025. arXiv:1807.06880 [gr-qc]

23. A. Cisterna, J. Oliva, Class. Quantum Gravity 35(3), 035012 (2018). arXiv:1708.02916 [hep-th]

24. A. Chamblin, S.W. Hawking, H.S. Reall, Phys. Rev. D 61, 065007 (2000). https://doi.org/10.1103/PhysRevD.61.065007. arXiv:hep-th/9909205

25. M. Astorino, F. Canfora, M. Lagos, A. Vera, Phys. Rev. D 97(12), 124032 (2018). https://doi.org/10.1103/PhysRevD.97. 124032. arXiv:1805.12252 [hep-th]

26. A. Cisterna, C. Corral, S. del Pino, arXiv:1809.02903 [gr-qc]

27. G.W. Horndeski, Int. J. Theor. Phys. 10, 363 (1974). https://doi. org/10.1007/BF01807638

28. A. Nicolis, R. Rattazzi, E. Trincherini, Phys. Rev. D 79, 064036 (2009). https://doi.org/10.1103/PhysRevD.79.064036. arXiv:0811.2197 [hep-th]

29. C. Deffayet, G. Esposito-Farese, A. Vikman, Phys. Rev. D 79, 084003 (2009). https://doi.org/10.1103/PhysRevD.79.084003. arXiv:0901.1314 [hep-th]

30. C. Deffayet, S. Deser, G. Esposito-Farese, Phys. Rev. D 80, 064015 (2009). https://doi.org/10.1103/PhysRevD.80.064015. arXiv:0906.1967 [gr-qc]

31. A. De Felice, T. Kobayashi, S. Tsujikawa, Phys. Lett. B 706, 123 (2011). https://doi.org/10.1016/j.physletb.2011.11.028. arXiv: 1108.4242 [gr-qc]

32. F. Piazza, F. Vernizzi, Class. Quantum Gravity 30, 214007 (2013). https://doi.org/10.1088/0264-9381/30/21/214007. arXiv: 1307.4350 [hep-th]

33. A. De Felice, S. Tsujikawa, JCAP 1202, 007 (2012). https://doi. org/10.1088/1475-7516/2012/02/007. arXiv:1110.3878 [gr-qc]

34. C. Germani, A. Kehagias, Phys. Rev. Lett. 105, 011302 (2010). https://doi.org/10.1103/PhysRevLett.105.011302. arXiv: 1003.2635 [hep-ph]

35. L. Amendola, Phys. Lett. B 301, 175 (1993). https://doi.org/10. 1016/0370-2693(93)90685-B. arXiv:gr-qc/9302010

36. S.V. Sushkov, Phys. Rev. D 80, 103505 (2009). https://doi.org/10. 1103/PhysRevD.80.103505. arXiv:0910.0980 [gr-qc]

37. R. Myrzakulov, L. Sebastiani, Astrophys. Space Sci. 361(2), 62 (2016). https://doi.org/10.1007/s10509-015-2646-3. arXiv: 1512.00402 [gr-qc]

38. J.P. Bruneton, M. Rinaldi, A. Kanfon, A. Hees, S. Schlogel, A. Fuzfa, Adv. Astron. 2012, 430694 (2012). https://doi.org/10.1155/ 2012/430694. arXiv:1203.4446 [gr-qc] 
39. M. Rinaldi, Phys. Rev. D 86, 084048 (2012). https://doi.org/10. 1103/PhysRevD.86.084048. arXiv:1208.0103 [gr-qc]

40. E. Babichev, C. Charmousis, JHEP 1408, 106 (2014). https://doi. org/10.1007/JHEP08(2014)106. arXiv:1312.3204 [gr-qc]

41. A. Anabalon, A. Cisterna, J. Oliva, Phys. Rev. D 89, 084050 (2014). https://doi.org/10.1103/PhysRevD.89.084050. arXiv:1312.3597 [gr-qc]

42. M. Minamitsuji, Phys. Rev. D 89, 064017 (2014). https://doi.org/ 10.1103/PhysRevD.89.064017. arXiv:1312.3759 [gr-qc]

43. T. Kobayashi, N. Tanahashi, PTEP 2014, 073E02 (2014). https:// doi.org/10.1093/ptep/ptu096. arXiv:1403.4364 [gr-qc]

44. A. Cisterna, C. Erices, Phys. Rev. D 89, 084038 (2014). https://doi. org/10.1103/PhysRevD.89.084038. arXiv:1401.4479 [gr-qc]

45. C. Charmousis, T. Kolyvaris, E. Papantonopoulos, M. Tsoukalas, JHEP 1407, 085 (2014). https://doi.org/10.1007/ JHEP07(2014)085. arXiv:1404.1024 [gr-qc]

46. A. Cisterna, T. Delsate, M. Rinaldi, Phys. Rev. D 92(4), 044050 (2015). arXiv:1504.05189 [gr-qc]

47. A. Cisterna, T. Delsate, L. Ducobu, M. Rinaldi, Phys. Rev. D 93(8), 084046 (2016). arXiv:1602.06939 [gr-qc]

48. D.G. Boulware, S. Deser, Phys. Rev. Lett. 55, 2656 (1985). https:// doi.org/10.1103/PhysRevLett.55.2656

49. R.M. Wald, Phys. Rev. D 48(8), R3427 (1993). https://doi.org/10. 1103/PhysRevD.48.R3427. arXiv:gr-qc/9307038

50. T. Jacobson, R.C. Myers, Phys. Rev. Lett. 70, 3684 (1993). https:// doi.org/10.1103/PhysRevLett.70.3684. arXiv:hep-th/9305016

51. G. Dotti, J. Oliva, R. Troncoso, Phys. Rev. D 75, 024002 (2007). https://doi.org/10.1103/PhysRevD.75.024002. arXiv:hep-th/0607062

52. "Chern-Simons (Super)Gravity", M. Hassaine, J. Zanelli, WSPC, (2016)

53. A. Anabalon, N. Deruelle, Y. Morisawa, J. Oliva, M. Sasaki, D. Tempo, R. Troncoso, Class. Quantum Gravity 26, 065002 (2009). https://doi.org/10.1088/0264-9381/26/6/065002. arXiv:0812.3194 [hep-th]

54. A. Anabalon, N. Deruelle, D. Tempo, R. Troncoso, Int. J. Mod. Phys. D 20, 639 (2011). https://doi.org/10.1142/ S0218271811018974. arXiv:1009.3030 [gr-qc]
55. M. Cvetic, X.H. Feng, H. Lu, C.N. Pope, Phys. Lett. B 765, 181 (2017). https://doi.org/10.1016/j.physletb.2016.12.018. arXiv:1609.09136 [hep-th]

56. G. Giribet, J. Oliva, R. Troncoso, JHEP 0605, 007 (2006). https:// doi.org/10.1088/1126-6708/2006/05/007. arXiv:hep-th/0603177

57. D. Kastor, R.B. Mann, JHEP 0604, 048 (2006). https://doi.org/10. 1088/1126-6708/2006/04/048. arXiv:hep-th/0603168

58. A. Giacomini, M. Lagos, J. Oliva, A. Vera, Phys. Rev. D 98(4), 044019 (2018). https://doi.org/10.1103/PhysRevD.98. 044019. arXiv:1804.03130 [hep-th]

59. D. Kastor, S. Ray, J. Traschen, Class. Quantum Gravity 34(19), 195005 (2017). https://doi.org/10.1088/1361-6382/ aa8608. arXiv: 1706.06684 [gr-qc]

60. T. Kobayashi, T. Tanaka, Phys. Rev. D 71, 084005 (2005). https:// doi.org/10.1103/PhysRevD.71.084005. arXiv:gr-qc/0412139

61. Y. Brihaye, T. Delsate, E. Radu, JHEP 1007, 022 (2010). https:// doi.org/10.1007/JHEP07(2010)022. arXiv:1004.2164 [hep-th]

62. C. Charmousis, M. Tsoukalas, Phys. Rev. D 92(10), 104050 (2015). https://doi.org/10.1103/PhysRevD.92.104050. arXiv:1506.05014 [gr-qc]

63. C. Gao, arXiv:1804.03535 [gr-qc]

64. A. Giacomini, J. Oliva, A. Vera, Phys. Rev. D 91(10), 104033 (2015). https://doi.org/10.1103/PhysRevD.91.104033. arXiv:1503.03696 [hep-th]

65. M. Lagos, J. Oliva A. Vera, Instability of black $p$-branes in Gauss-Bonnet theory in ten dimensions. https://doi.org/10.1142/ 9789813226609_0177

66. A. Giacomini, C. Henrquez-Bez, M. Lagos, J. Oliva, A. Vera, Phys. Rev. D 93(10), 104005 (2016). https://doi.org/10.1103/PhysRevD. 93.104005. arXiv:1603.02670 [hep-th]

67. Y. Hyakutake, JHEP 1509, 067 (2015). https://doi.org/10.1007/ JHEP09(2015)067. arXiv:1503.05083 [gr-qc]

68. R. Emparan, R. Suzuki, K. Tanabe, JHEP 1306, 009 (2013). https:// doi.org/10.1007/JHEP06(2013)009. arXiv:1302.6382 [hep-th]

69. B. Chen, P.C. Li, C.Y. Zhang, JHEP 1710, 123 (2017). https://doi. org/10.1007/JHEP10(2017)123. arXiv:1707.09766 [hep-th]

70. D. Lovelock, J. Math. Phys. 12, 498 (1971) 